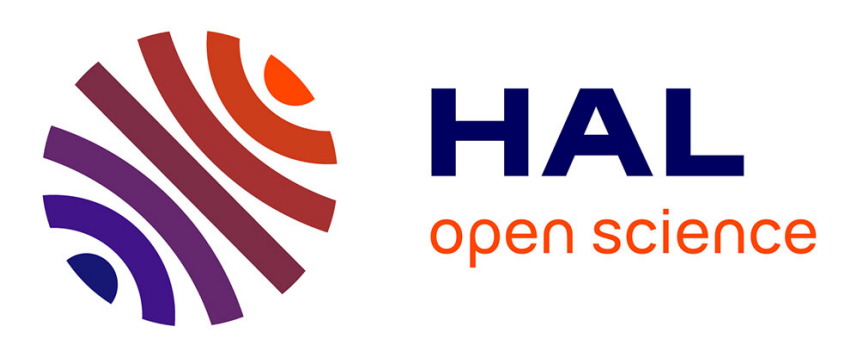

\title{
Denotational semantics for programming languages, balanced quasi-metrics and fixed points (SI-CMMSE2006)
}

\author{
Oscar Valero, Jesus Rodriguez-Lopez, Salvador Romaguera
}

\section{To cite this version:}

Oscar Valero, Jesus Rodriguez-Lopez, Salvador Romaguera. Denotational semantics for programming languages, balanced quasi-metrics and fixed points (SI-CMMSE2006). International Journal of Computer Mathematics, 2008, 85 (03-04), pp.623-630. 10.1080/00207160701210653 . hal-00545349

\author{
HAL Id: hal-00545349 \\ https://hal.science/hal-00545349
}

Submitted on 10 Dec 2010

HAL is a multi-disciplinary open access archive for the deposit and dissemination of scientific research documents, whether they are published or not. The documents may come from teaching and research institutions in France or abroad, or from public or private research centers.
L'archive ouverte pluridisciplinaire HAL, est destinée au dépôt et à la diffusion de documents scientifiques de niveau recherche, publiés ou non, émanant des établissements d'enseignement et de recherche français ou étrangers, des laboratoires publics ou privés. 


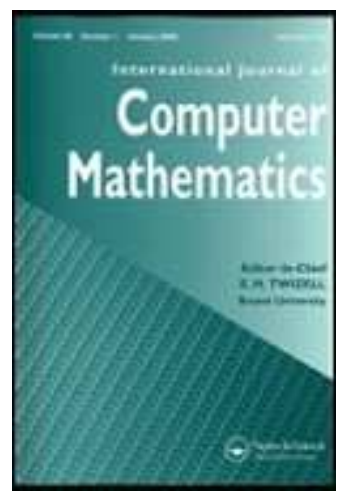

\section{Denotational semantics for programming languages, balanced quasi-metrics and fixed points (SI-CMMSE2006)}

\begin{tabular}{|c|c|}
\hline Journal: & International Journal of Computer Mathematics \\
\hline Manuscript ID: & GCOM-2006-0163.R1 \\
\hline Manuscript Type: & Original Article \\
\hline $\begin{array}{r}\text { Date Submitted by the } \\
\text { Author: }\end{array}$ & 02-Jan-2007 \\
\hline Complete List of Authors: & $\begin{array}{l}\text { VALERO, OSCAR; Universidad de las Islas Baleares, Departamento } \\
\text { de Ciencias Matematicas e Informatica } \\
\text { Rodriguez-Lopez, Jesus; Universidad Politecnica de Valencia, } \\
\text { Matematica Aplicada } \\
\text { Romaguera, Salvador; Universidad Politecnica de Valencia, } \\
\text { Matematica Aplicada }\end{array}$ \\
\hline Keywords: & $\begin{array}{l}\text { domain of words, Baire metric, balanced quasi-metric, algorithm, } \\
\text { recurrence equation }\end{array}$ \\
\hline
\end{tabular}

\section{今 scholarONE" \\ Manuscript Central}




\title{
Denotational semantics for programming languages, balanced quasi-metrics and fixed points
}

\author{
J. Rodríguez-López†, S. Romaguera†, O. Valeroł* \\ †Departamento de Matemática Aplicada, Universidad Politécnica de Valencia, 46071, Valencia, Spain \\ $\ddagger$ Departamento de Matemáticas e Informática, Universidad de las Islas Baleares, 07122, Baleares, Spain
}

\begin{abstract}
A new mathematical model is introduced for the study of the domain of words. We do it by means of the introduction of a suitable balanced quasi-metric on the set of all words over an alphabet. It will be shown that this construction has better quasi-metric and topological properties than several classical constructions. We also prove a fixed point theorem which allows us to develop an application for the study of Probabilistic Divide and Conquer Algorithms.
\end{abstract}

Key words: domain of words, Baire metric, balanced quasi-metric, algorithm, recurrence equation

MSC 2000: 68Q55, 68Q25, 54E35

\section{Introduction}

It is well known that the classical Baire metric (or Baire distance) provides an efficient tool to obtain denotational models for programming languages (see [3], [4], [5]). It is also applied, for instance, to study the representation

\footnotetext{
${ }^{*}$ Corresponding author. Email: o.valero@uib.es.

The authors thank the support of the Spanish Ministry of Education and Science, and FEDER, grant MTM2006-14925-C02-01
} 
of real numbers by means of regular languages [17] and in modelling streams of information in parallel computation [13]. The Baire metric is especially useful for the measurement of distances between totally defined objects (infinite words), and, hence, those computational processes where it is necessary to take into account the information given by the partially defined objects (finite words) are usually modelled by using Baire quasi-pseudo-metrics (see [2], [18], [19], [28], [29], [25], [27], etc.). However, these quasi-pseudo-metrics generally induce only $T_{0}$ topologies. Motivated, in part, by this fact, in Section 2 we present a new model for the measurement of distances between words by means of the notion of a balanced quasi-metric. Moreover, our model possesses other rich quasi-metric and topological properties such as right K-completeness, Hausdorffness and uniformizability -equivalently, complete regularity- and it respects several essential aspects of the computational interpretation given by other known Baire quasi-pseudo-metrics. An application of our approach to the analysis of Probabilistic Divide and Conquer Algorithms via techniques of fixed point is also given.

Our basic references for quasi-pseudo-metric spaces are [10] and [16] and for topology is [8].

Let us recall that a quasi-pseudo-metric on a set $X$ is a nonnegative real valued function $d$ on $X \times X$ such that for all $x, y, z \in X:$ (i) $d(x, x)=0$; (ii) $d(x, y) \leq d(x, z)+d(z, y)$.

By a quasi-metric on $X$ we mean a quasi-pseudo-metric $d$ on $X$ that satisfies the following condition: (iii) $d(x, y)=0$ if and only if $x=y$.

A quasi-(pseudo-)metric space is a pair $(X, d)$ such that $X$ is a (nonempty) set and $d$ is a quasi-(pseudo-)metric on $X$.

Each quasi-pseudo-metric $d$ on $X$ generates a topology $\tau_{d}$ on $X$ which has as a base the family of open $d$-balls $\left\{B_{d}(x, r): x \in X, r>0\right\}$, where $B_{d}(x, r)=\{y \in X: d(x, y)<r\}$ for all $x \in X$ and $r>0$.

Observe that if $d$ is a quasi-metric, then $\tau_{d}$ is a $T_{1}$ topology.

Given a quasi-(pseudo-)metric $d$ on $X$, then the function $d^{-1}$ defined on $X \times X$ by $d^{-1}(x, y)=d(y, x)$, is also a quasi-(pseudo-)metric on $X$, called the conjugate of $d$. Finally, the function $d^{s}$ defined on $X \times X$ by $d^{s}(x, y)=\max \left\{d(x, y), d^{-1}(x, y)\right\}$ is a (pseudo-)metric on $X$. 


\section{The Baire balanced quasi-metric on the do- main of words}

Let $\Sigma$ be a nonempty alphabet. Denote by $\Sigma^{F}$ the set of finite sequences ("finite words") over $\Sigma$ and by $\Sigma^{\infty}$ the set of finite and infinite sequences over $\Sigma$. We assume that the empty sequence $\varnothing$ is an element of $\Sigma^{F}$.

Denote by $\sqsubseteq$ the prefix order on $\Sigma^{\infty}$, i.e. $x \sqsubseteq y \Leftrightarrow x$ is a prefix of $y$. If $x \sqsubseteq y$ with $x \neq y$ we write $x \sqsubset y$.

For each $x, y \in \Sigma^{\infty}$ we define $x \sqcap y$ as the longest common prefix of $x$ and $y$, and for each $x \in \Sigma^{\infty}$ we denote by $\ell(x)$ the length of $x$. Then $\ell(x) \in[1, \infty]$ whenever $x \neq \varnothing$ and $\ell(\varnothing)=0$.

Remark 1. If $\left(x_{n}\right)_{n \in \mathbb{N}}$ is a strictly increasing sequence in $\Sigma^{\infty}$ with respect to $\sqsubseteq$, i.e. $x_{n} \sqsubset x_{n+1}$ for all $n \in \mathbb{N}$, we shall denote by $\sqcup_{k} x_{k}$ the unique element in $\Sigma^{\infty}$ such that $x_{n} \sqcap\left(\sqcup_{k} x_{k}\right)=x_{n}$ for all $n \in \mathbb{N}$. Note that, in this case, $x_{n} \in \Sigma^{F}$ for all $n \in \mathbb{N}$ and $\ell\left(\sqcup_{k} x_{k}\right)=\lim _{n \rightarrow \infty} \ell\left(x_{n}\right)=\infty$.

Let us recall that the Baire metric (or Baire distance) on $\Sigma^{\infty}$ is the metric $d_{B}$ defined by

$$
\begin{aligned}
& d_{B}(x, x)=0 \quad \text { for all } x \in \Sigma^{\infty}, \\
& d_{B}(x, y)=2^{-\ell(x \sqcap y)} \text { for all } x, y \in \Sigma^{\infty} \text { with } x \neq y .
\end{aligned}
$$

(We adopt the convention that $2^{-\infty}=0$ ).

Smyth introduced in [28] the following quasi-pseudo-metric modification of the Baire metric. For each $x, y \in \Sigma^{\infty}$ let

$$
\begin{aligned}
& d_{\sqsubseteq}(x, y)=0 \quad \text { if } x \sqsubseteq y, \\
& d_{\sqsubseteq}(x, y)=d_{B}(x, y) \quad \text { otherwise. }
\end{aligned}
$$
on $\Sigma^{\infty}$.

Then $d_{\sqsubseteq}$ is a $T_{0}$ quasi-pseudo-metric on $\Sigma^{\infty}$ and $\left(d_{\sqsubseteq}\right)^{s}$ is the Baire metric

Observe that condition $d_{\sqsubseteq}(x, y)=0$ can be used to distinguish between the case that $x$ is a prefix of $y$ and the remaining cases for $x, y \in \Sigma^{\infty}$.

Other $T_{0}$ quasi-pseudo-metric variants of the Baire metric, with applications, may be found in [15], [18], [19], [22], [25], etc. However, it is well known and easy to see that both $d_{\sqsubseteq}$ and these quasi-pseudo-metrics do not generate Hausdorff (in fact, not even $T_{1}$ ) topologies. Next we construct a new quasi-metric on $\Sigma^{\infty}$ which possesses several interesting properties. In 
particular, the generated topology will be Hausdorff and completely regular. Furthermore, the information given by condition $d_{\sqsubseteq}(x, y)=0$ is also preserved by our approach.

Doitchinov introduced in [6] the notion of a balanced quasi-metric space in order to obtain a consistent theory of quasi-metric completion that preserves complete regularity.

Recall that a quasi-metric $d$ on a set $X$ is balanced provided that whenever $\left(x_{n}\right)_{n \in \mathbb{N}}$ and $\left(y_{n}\right)_{n \in \mathbb{N}}$ are sequences in $X$ and $x, y$ are points in $X$ such that $d\left(x, x_{n}\right) \leq r_{1}, d\left(y_{m}, y\right) \leq r_{2}$ for all $n, m \in \mathbb{N}$, and $\lim _{n, m \rightarrow \infty} d\left(y_{m}, x_{n}\right)=0$, then $d(x, y) \leq r_{1}+r_{2}$. A quasi-metric space $(X, d)$ is called balanced if $d$ is a balanced quasi-metric on $X$. Each balanced quasi-metric space is Hausdorff and completely regular $[6]$.

Remark 2. It is well known and easy to see that a quasi-metric space $(X, d)$ is balanced if and only if $\left(X, d^{-1}\right)$ is balanced. Therefore, if $(X, d)$ is balanced, then $\left(X, d^{-1}\right)$ is Hausdorff and completely regular.

Theorem 1. Let $\Sigma$ be an alphabet and let $q_{b}: \Sigma^{\infty} \times \Sigma^{\infty} \rightarrow[0,1]$ be the function given by

$$
\begin{aligned}
& q_{b}(x, y)=2^{-\ell(x)}-2^{-\ell(y)} \quad \text { if } x \sqsubseteq y, \\
& q_{b}(x, y)=1 \quad \text { otherwise. }
\end{aligned}
$$

Then, the following statements hold:

(1) $q_{b}$ is a balanced quasi-metric on $\Sigma^{\infty}$.

(2) $\lim _{n \rightarrow \infty} q_{b}\left(x_{n}, x\right)=0 \Rightarrow \lim _{n \rightarrow \infty} d_{B}\left(x_{n}, x\right)=0$.

(3) If $\left(x_{n}\right)_{n \in \mathbb{N}}$ is a strictly increasing sequence in $\Sigma^{\infty}$ with respect to $\sqsubseteq$, then $\lim _{n \rightarrow \infty} q_{b}\left(x_{n}, \sqcup_{k} x_{k}\right)=0$.

Proof. (1) We first show that $q_{b}$ is a quasi-metric on $\Sigma^{\infty}$.

It is clear that for $x, y \in \Sigma^{\infty}$ such that $q_{b}(x, y)=0$, it follows that $x=y$.

Now let $x, y, z \in \Sigma^{\infty}$. If $q_{b}(x, z)=1$ or $q_{b}(z, y)=1$, it is obvious that $q_{b}(x, y) \leq q_{b}(x, z)+q_{b}(z, y)$. Assume then that $q_{b}(x, z)<1$ and $q_{b}(z, y)<1$. Thus $x \sqsubseteq z$ and $z \sqsubseteq y$, so $x \sqsubseteq y$. Hence

$$
\begin{aligned}
q_{b}(x, y) & =2^{-\ell(x)}-2^{-\ell(y)}=2^{-\ell(x)}-2^{-\ell(z)}+2^{-\ell(z)}-2^{-\ell(y)} \\
& =q_{b}(x, z)+q_{b}(z, y) .
\end{aligned}
$$

We have shown that $q_{b}$ is a quasi-metric on $\Sigma^{\infty}$. 
In order to prove that $q_{b}$ is balanced, let $\left(x_{n}\right)_{n \in \mathbb{N}}$ and $\left(y_{n}\right)_{n \in \mathbb{N}}$ be sequences in $\Sigma^{\infty}$, and $x, y$ points in $\Sigma^{\infty}$ such that $q_{b}\left(x, x_{n}\right) \leq r_{1}$ for all $n \in \mathbb{N}$, $q_{b}\left(y_{m}, y\right) \leq r_{2}$ for all $m \in \mathbb{N}$, and $\lim _{n, m \rightarrow \infty} q_{b}\left(y_{m}, x_{n}\right)=0$. We wish to show that $q_{b}(x, y) \leq r_{1}+r_{2}$. If $r_{1}=1$ or $r_{2}=1$, obviously $q_{b}(x, y) \leq r_{1}+r_{2}$. Then, we suppose that $r_{1}<1$ and $r_{2}<1$. Thus $x \sqsubseteq x_{n}$ for all $n \in \mathbb{N}$, and $y_{m} \sqsubseteq y$ for all $m \in \mathbb{N}$.

We shall distinguish two cases: i) $\ell(x)=\infty$, ii) $\ell(x)<\infty$.

In case i), from the fact that $x \sqsubseteq x_{n}$, it follows that $x=x_{n}$ for all $n \in \mathbb{N}$. Thus $\lim _{m \rightarrow \infty} q_{b}\left(y_{m}, x\right)=0$, and hence $\lim _{m \rightarrow \infty} \ell\left(y_{m}\right)=\infty$. Since $y_{m} \sqsubseteq y$ for all $m \in \mathbb{N}$, it follows that $\ell(y)=\infty$. Therefore

$$
\lim _{m \rightarrow \infty} d_{B}\left(x, y_{m}\right)=\lim _{m \rightarrow \infty} d_{B}\left(y, y_{m}\right)=0
$$

so that $x=y$, i.e. $q_{b}(x, y)=0$.

In case ii), since $\lim _{n, m \rightarrow \infty} q_{b}\left(y_{m}, x_{n}\right)=0$, there is $n_{0} \in \mathbb{N}$ such that for each $n, m \geq n_{0}, q_{b}\left(y_{m}, x_{n}\right)<1$, so that $y_{m} \sqsubseteq x_{n}$ whenever $n, m \geq n_{0}$. Assume first that $\ell\left(y_{m}\right)<\ell(x)$ for all $m \geq n_{0}$. Since there is $k \geq n_{0}$ such that

$$
q_{b}\left(y_{k}, x_{k}\right)<2^{-\ell(x)}
$$

we deduce that

$$
\begin{aligned}
2^{-\ell\left(y_{k}\right)} & <2^{-\ell\left(x_{k}\right)}+2^{-\ell(x)} \\
& \Rightarrow 2^{\ell(x)}<2^{\ell\left(y_{k}\right)}\left[2^{\ell(x)-\ell\left(x_{k}\right)}+1\right] \\
& \Rightarrow 2^{\ell(x)}<2^{\ell\left(y_{k}\right)+1} \\
& \Rightarrow \ell(x)<\ell\left(y_{k}\right)+1
\end{aligned}
$$

which provides a contradiction. Consequently, there is $m_{0} \geq n_{0}$ with $\ell(x) \leq$ $\ell\left(y_{m_{0}}\right)$; but $x \sqsubseteq x_{m_{0}}$ and $y_{m_{0}} \sqsubseteq x_{m_{0}}$, so we deduce that $x \sqsubseteq y_{m_{0}}$, and hence

$$
q_{b}\left(x, y_{m_{0}}\right)=2^{-\ell(x)}-2^{-\ell\left(y_{m_{0}}\right)} \leq 2^{-\ell(x)}-2^{-\ell\left(x_{m_{0}}\right)}=q_{b}\left(x, x_{m_{0}}\right) .
$$

Therefore

$$
\begin{aligned}
q_{b}(x, y) & \leq q_{b}\left(x, y_{m_{0}}\right)+q_{b}\left(y_{m_{0}}, y\right) \\
& \leq q_{b}\left(x, x_{m_{0}}\right)+q_{b}\left(y_{m_{0}}, y\right) \leq r_{1}+r_{2} .
\end{aligned}
$$

We conclude that the quasi-metric $q_{b}$ is balanced. 
(2) Let $\left(x_{n}\right)_{n \in \mathbb{N}}$ be a sequence in $\Sigma^{\infty}$ and $x \in \Sigma^{\infty}$ such that $\lim _{n \rightarrow \infty} q_{b}\left(x_{n}, x\right)=$ 0 . If $x \in \Sigma^{F}$, then $x_{n}=x$ eventually. If $\ell(x)=\infty$, then there is $n_{0} \in \mathbb{N}$ such that

$$
q_{b}\left(x_{n}, x\right)=2^{-\ell\left(x_{n}\right)}=2^{-\ell\left(x_{n} \sqcap x\right)}=d_{B}\left(x_{n}, x\right),
$$

for all $n \geq n_{0}$. Consequently $\lim _{n \rightarrow \infty} d_{B}\left(x_{n}, x\right)=0$.

(3) If $\left(x_{n}\right)_{n \in \mathbb{N}}$ is a strictly increasing sequence in $\Sigma^{\infty}$ with respect to $\sqsubseteq$, then $x_{n} \sqsubset x_{n+1}$ for all $n \in \mathbb{N}$, and $\sqcup_{k} x_{k}$ the unique element in $\Sigma^{\infty}$ such that $x_{n} \sqcap\left(\sqcup_{k} x_{k}\right)=x_{n}$ for all $n \in \mathbb{N}$. Hence $x_{n} \in \Sigma^{F}$ for all $n \in \mathbb{N}$ and $\ell\left(\sqcup_{k} x_{k}\right)=\lim _{n \rightarrow \infty} \ell\left(x_{n}\right)=\infty$ (see Remark 1). Therefore

$$
q_{b}\left(x_{n}, \sqcup_{k} x_{k}\right)=2^{-\ell\left(x_{n}\right)}-2^{-\ell\left(\sqcup_{k} x_{k}\right)}=2^{-\ell\left(x_{n}\right)},
$$

for all $n \in \mathbb{N}$, and thus, $\lim _{n \rightarrow \infty} q_{b}\left(x_{n}, x\right)=0$.

Remark 3. It is clear that the measurement of the distance from a word $x$ to another word $y$ by means of the Baire metric does not permit us to know if $x$ is a prefix of $y$ or not. This inconvenience is saved by using the Baire quasi-pseudo-metric $d_{\sqsubseteq}$. However, if $x, y, z \in \Sigma^{\infty}$ satisfy $x \sqsubset y \sqsubset z$, one obtains $d_{\sqsubseteq}(x, z)=d_{\sqsubseteq}(y, z)=0$, and we can not decide which word of the two, $x$ or $y$, gives a better approximation of $z$. For instance, if we consider the totally defined object $\pi$ and the partially defined ones $x=3.1415$ and $y=3.141592$, then it is clear that $y$ contains more information on $\pi$ than $x$, but the quasi-metric $d_{\sqsubseteq}$ is not sensitive to this amount of information. The quasi-metric $q_{b}$ avoids this disadvantage because $q_{b}(3.1415, \pi)=2^{-5}$ and $q_{b}(3.141592, \pi)=2^{-7}$ as is desirable. In general, for $x, y, z \in \Sigma^{\infty}$ with $x \sqsubset y \sqsubset z$, we have $\ell(x)<\ell(y)<\ell(z)$ and thus $q_{b}(x, z)>q_{b}(y, z)$. This last inequality allows the word with smaller information $x$ to be distinguished from the word $y$ which captures greater information of the derived one from $z$. Furthermore $q_{b}(x, y)<1$ if and only if $x \sqsubseteq y$ with $x \neq \varnothing$. Other useful properties of $q_{b}$ and $\left(q_{b}\right)^{-1}$ are given in Remark 4 (c) and Theorem 2 below.

Remark 4. (a) We first note that Theorem 1(1) and Remark 2 imply that both $\left(\Sigma^{\infty}, q_{b}\right)$ and $\left(\Sigma^{\infty},\left(q_{b}\right)^{-1}\right)$ are Hausdorff and completely regular (actually $\left(q_{b}\right)^{-1}$ is also a balanced quasi-metric). In fact, topological properties of $\left(q_{b}\right)^{-1}$ are used in Theorem 4 below.

(b) From Theorem 1(2) it immediately follows that $\tau_{\left(q_{b}\right)^{-1}}$ is finer than $\tau_{d_{B}}$, i.e. $\tau_{d_{B}} \subseteq \tau_{\left(q_{b}\right)^{-1}}$, and thus $\left(\Sigma^{\infty}, \tau_{\left(q_{b}\right)^{-1}}\right)$ is submetrizable. 
(c) Theorem 1(3) provides an appropriate computational interpretation of the fact that each infinite word (totally defined object) can be expressed as the supremum of a strictly increasing sequence of finite words (partially defined objects) and it captures the notion of the amount of information defined by such a sequence. This fact can be mathematically modelled in the realm of right K-sequentially complete quasi-metric spaces as we show in the following.

Let us recall [20], that a sequence $\left(x_{n}\right)_{n \in \mathbb{N}}$ in a quasi-metric space $(X, d)$ is right K-Cauchy if for each $\varepsilon>0$ there is $n_{0} \in \mathbb{N}$ such that $d\left(x_{m}, x_{n}\right)<\varepsilon$ whenever $m \geq n \geq n_{0}$. The quasi-metric space $(X, d)$ is said to be right $\mathrm{K}$-sequentially complete if every right $\mathrm{K}$-Cauchy sequence is convergent with respect to $\tau_{d}$.

We point out that right K-sequential completeness provides an efficient tool for the study of function spaces and hyperspaces for quasi-metric spaces (see, for instance, Section 9 of [16]).

Theorem 2. The quasi-metric space $\left(\Sigma^{\infty},\left(q_{b}\right)^{-1}\right)$ is right K-sequentially complete.

Proof. Let $\left(x_{n}\right)_{n \in \mathbb{N}}$ be a right K-Cauchy sequence in $\left(\Sigma^{\infty},\left(q_{b}\right)^{-1}\right)$. Assume without loss of generality that this sequence is strictly increasing with respect to $\sqsubseteq$. It then follows from Theorem 1 (3) that $\lim _{n \rightarrow \infty} q_{b}\left(x_{n}, \sqcup_{k} x_{k}\right)=0$. Therefore $\left(\Sigma^{\infty},\left(q_{b}\right)^{-1}\right)$ is right K-sequentially complete.

\section{A fixed point theorem on $\left(\Sigma^{\infty},\left(q_{b}\right)^{-1}\right)$ with application to the complexity analysis of algorithms}

In this section we obtain a fixed point theorem for certain self-maps on the balanced right K-sequentially complete quasi-metric space $\left(\Sigma^{\infty},\left(q_{b}\right)^{-1}\right)$. We apply such a result to deduce that for several recurrence equations based on the recursive structure of Probabilistic Divide and Conquer Algorithm [9], the associated functionals have a unique fixed point which is the solution for the corresponding recurrence equation. However, in the case that our self-map $f$ satisfies the condition that $f x$ is not a prefix of $f y, f$ cannot be 
a contraction on $\Sigma^{\infty}$ because $q_{b}(x, y)=1$ whenever $x$ is not a prefix of $y$. For this reason we will establish our result only assuming that the self-map satisfies a contraction property on the orbit of a point of $\Sigma^{\infty}$, which will be sufficient for our purposes in this context. Actually, we will prove a more general result in the framework of Hausdorff right K-sequentially complete quasi-metric spaces.

If $(X, d)$ is a quasi-metric space we say that a self-map $f: X \rightarrow X$ is continuous if it is continuous from $\left(X, \tau_{d}\right)$ to $\left(X, \tau_{d}\right)$.

Theorem 3. Let $(X, d)$ be a Hausdorff right K-sequentially complete quasimetric space and let $f: X \rightarrow X$ be a continuous self-map. If there are $x_{0} \in X$ and $k \in(0,1)$ such that

$$
d\left(f^{n+1} x_{0}, f^{n} x_{0}\right) \leq k d\left(f^{n} x_{0}, f^{n-1} x_{0}\right)
$$

for all $n \in \mathbb{N}$, then $f$ has a fixed point.

Proof. By our contraction condition, for each $n \in \mathbb{N}$ we obtain

$$
d\left(f^{n+1} x_{0}, f^{n} x_{0}\right) \leq k^{n} d\left(f x_{0}, x_{0}\right)
$$

By the triangle inequality it easily follows that for each $m, n \in \mathbb{N}$,

$$
d\left(f^{n+m} x_{0}, f^{n} x_{0}\right) \leq \frac{k^{n}}{1-k} d\left(f x_{0}, x_{0}\right),
$$

and, consequently $\left(f^{n} x_{0}\right)_{n \in \mathbb{N}}$ is a right K-Cauchy sequence. Thus there is $y \in X$ such that $\lim _{n \rightarrow \infty} d\left(y, f^{n} x_{0}\right)=0$. By continuity of $f$ it follows that $\lim _{n \rightarrow \infty} d\left(f y, f^{n+1} x_{0}\right)=0$, so $y=f y$ by Hausdorffness of $(X, d)$. This concludes the proof.

The following easy example shows that uniqueness of the fixed point in the preceding theorem does not hold, in general.

Example. Let $X=a, b$ and let $d$ be the discrete metric on $X$. Define $f(a)=a$ and $f(b)=b$, and note that the inequality $(\star)$ is obviously satisfied both for $a$ and $b$.

Now suppose that $\Sigma$ is an alphabet and let $q_{b}$ the balanced quasi-metric on $\Sigma^{\infty}$ constructed in Theorem 1 . Then $\left(\Sigma^{\infty},\left(q_{b}\right)^{-1}\right)$ is a right K-sequentially 
complete balanced (hence, Hausdorff) quasi-metric space by Theorem 2 and Remark 4 (a). Hence, we derive from Theorem 3 the following.

Theorem 4. Let $\Phi: \Sigma^{\infty} \rightarrow \Sigma^{\infty}$ be a continuous self-map on $\left(\Sigma^{\infty},\left(q_{b}\right)^{-1}\right)$. If there are $w_{0} \in \Sigma^{\infty}$ and $k \in(0,1)$ such that

$$
q_{b}\left(\Phi^{n} w_{0}, \Phi^{n+1} w_{0}\right) \leq k q_{b}\left(\Phi^{n-1} w_{0}, \Phi^{n} w_{0}\right),
$$

for all $n \in \mathbb{N}$, then $\Phi$ has a fixed point.

When discussing the analysis of Probabilistic Divide and Conquer Algorithms by means of recurrences, the following general recurrence equation is obtained (see, for instance, Section 4 of [9]):

$$
T(n)=p(n)+\sum_{k=1}^{n-1} q(n, k) T(k),
$$

for $n \geq 2$, where $T(1) \geq 0, p(n)>0$, and $q(n, k)>0$ is proportional to the splitting probabilities that express the changes that a task of size $n$ involve a subtask of size $k<n$.

A paradigmatic example of the above recurrence equation is the sorting algorithm used in the Unix system. The average case analysis of this algorithm is discussed in [14], where the following recurrence equation is obtained:

$$
\begin{aligned}
& T(1)=0, \quad \text { and } \\
& T(n)=\frac{2(n-1)}{n}+\frac{n+1}{n} T(n-1), \quad n \geq 2 .
\end{aligned}
$$

We conclude the paper by applying Theorem 4 to prove that each recurrence equation of type $(*)$ has a unique solution.

To this end, consider as an alphabet $\Sigma$ the set of nonnegative real numbers. Let $T$ be a recurrence equation of type $(*)$. We associate to $T$ the functional $\Phi_{T}: \Sigma^{\infty} \rightarrow \Sigma^{\infty}$ given by $\left(\Phi_{T} w\right)_{1}=T(1)$ and

$$
\left(\Phi_{T} w\right)_{n}=p(n)+\sum_{k=1}^{n-1} q(n, k) w_{k}
$$

for all $n \geq 2$ (if $w \in \Sigma^{\infty}$ has length $n<\infty$, we write $w:=w_{1} w_{2} \ldots w_{n}$, and if $w$ is an infinite word we write $\left.w:=w_{1} w_{2} \ldots\right)$. 
It is clear by the construction that if $\ell(w)=n$ then $\ell\left(\Phi_{T} w\right)=n+1$ (in particular, $\ell\left(\Phi_{T} w\right)=\infty$ whenever $\left.\ell(w)=\infty\right)$.

Furthermore, we obtain

$$
(* *) \quad v \sqsubseteq w \Rightarrow \Phi_{T} v \sqsubseteq \Phi_{T} w .
$$

So for $v \sqsubseteq w$ with $\ell(w)=\infty$ we deduce

$$
q_{b}\left(\Phi_{T} v, \Phi_{T} w\right)=2^{-\ell\left(\Phi_{T} v\right)} \leq 2^{-\ell(v)},
$$

which implies that $\Phi_{T}$ is continuous from $\left(\Sigma^{\infty},\left(q_{b}\right)^{-1}\right)$ into itself, because if $\lim _{n \rightarrow \infty}\left(q_{b}\right)^{-1}\left(w, v_{n}\right)=0$, with $w \neq v_{n}$, then $\ell(w)=\infty$ and $\lim _{n \rightarrow \infty} \ell\left(v_{n}\right)=$ $\infty$, so $\lim _{n \rightarrow \infty}\left(q_{b}\right)^{-1}\left(\Phi_{T} w, \Phi_{T} v_{n}\right)=0$.

Now let $w$ be the element of $\Sigma^{\infty}$ given by $w:=T(1)$. Then $\ell(w)=1$. Since $w \sqsubseteq \Phi_{T} w$, it follows from condition (**) that

$$
\begin{aligned}
q_{b}\left(\Phi_{T}^{n} w, \Phi_{T}^{n+1} w\right) & =2^{-\ell\left(\Phi_{T}^{n} w\right)}-2^{-\ell\left(\Phi_{T}^{n+1} w\right)} \\
& =2^{-1}\left(2^{-\ell\left(\Phi_{T}^{n-1} w\right)}-2^{-\ell\left(\Phi_{T}^{n} w\right)}\right)=2^{-1} q_{b}\left(\left(\Phi_{T}^{n-1} w, \Phi_{T}^{n} w\right),\right.
\end{aligned}
$$

for all $n \in \mathbb{N}$. Consequently, we can apply Theorem 4 (with $k=1 / 2$ ), and thus $\Phi_{T}$ has a fixed point $w^{\prime}$ which is clearly unique by the construction of $\Phi_{T}$. We conclude that $w^{\prime}$ is the unique solution of the recurrence $T$.

Acknowledgement. The authors thank the referees for their valuable suggestions.

\section{References}

[1] V. Aho, J. Hopcroft And J. Ullman, Data Structures and Algorithms, Addison-Wesley, 1987.

[2] F. G. Arenas, M. L. Puertas and S. Romaguera, Ordered fractal semigroups as a model of computation, Math. Comput. Model. 36 (2002), 1121-1129.

[3] J. W. De Bakker And E. P. De Vink, Control Flow Semantics, The MIT Pres, Cambridge, Massachusetts, 1996.

[4] J. W. DE Bakker And E. P. DE Vink, A metric approach to control flow semantics, in: Proc. Eleventh Summer Conference on General Topology and Applications, Ann. New York Acad. Sci. 806 (1996), 1127. 
[5] J. W. DE BAKker And E. P. DE Vink, Denotational models for programming languages: applications of Banach's fixed point theorem, Topology Appl. 85 (1998), 35-52.

[6] D. Doitchinov, On completeness in quasi-metric spaces, Topology Appl. 30 (1988), 127-148.

[7] A. E. EMerson And C. S. Jutla, The complexity of tree automata and logic of programs, SIAM J. Comput. 29 (1999), 132-158.

[8] R. Engelking, General Topology, Polish Sci. Publ., Warsaw 1977.

[9] P. Flajolet, Analytic analysis of algorithms, in: ICALP'92, Vienna, July 1992; Automata, Languages and Programming, Lecture Notes in Computer Science 623, W. Kuich editor (1992), pp. 186-210.

[10] P. Fletcher And W. Lindgren, Quasi-Uniform Spaces, Marcel Dekker, 1982.

[11] L. M. García-Raffi, S. Romaguera and E. A. Sánchez-PÉrez, Sequence spaces and asymmetric norms in the theory of computational complexity, Math. Comput. Model. 36 (2002), 1-11.

[12] L. M. García-Raffi, S. Romaguera, E. A. SÁnchez-PÉrez And O. VALERO, Normed semialgebras: a mathematical model for the complexity analysis of programs and algorithms, in: Proc. 7th World Multiconference on Systemics, Cybernetics and Informatics, July 2003, Orlando, Florida, Vol. II, Computer Science and Engineering, pp. 55-58.

[13] G. Kahn, The semantics of a simple language for parallel processing, in: Proc. IFIP Congresss, Elsevier and North-Holland, Amsterdam 1974, pp. $471-475$.

[14] R. Kruse, Data Structures and Program Design, Prentice-Hall, Inc., 1984.

[15] H. P. A. KünzI, Nonsymmetric topology, Bolyai Soc. Math. Stud. 4, Topology, Szekszárd, 1993, Hungary, (Budapest 1995), 303-338.

[16] H. P. A. KüNZI, Nonsymmetric distances and their associated topologies: About the origin of basic ideas in the area of asymmetric topology, 
in: Handbook of the History of General Topology, Volume 3 (C. E. Aull and R. Lowen eds.), Kluwer, Dordrecht (2001), pp. 853-968.

[17] P. Lecomte And M. Rigo, On the representation of real numbers using regular languages, Theory Comput. Systems 35 (2002), 13-38.

[18] S. G. Matthews, Partial metric topology, in: Proc. 8th Summer Conference on General Topology and Applications, Ann. New York Acad. Sci. 728 (1994), 183-197.

[19] J. PIN, Uniformities on free semigroups, Internat. J. Algebra Comput. 9 (1999), 431-453.

[20] I. L. Reilly, P. V. Subrhamanyam and M. K. Vamanamurthy, Cauchy sequences in quasi-metric spaces, Monaths. Math. 93 (1982), 127-140.

[21] S. Romaguera, E. A. SÁnchez-PÉrez and O. Valero, Computing complexity distances between algorithms, Kybernetika 39 (2003), 569582.

[22] S. Romaguera And M. SAnchis, Applications of utility functions defined on quasi-metric spaces, J. Math. Anal. Appl. 283 (2003), 219235.

[23] S. Romaguera and M. Schellekens, Quasi-metric properties of complexity spaces, Topology Appl. 98 (1999), 311-322.

[24] S. Romaguera And M. Schellekens, Duality and quasi-normability for complexity spaces, Appl. Gen. Topology 3 (2002), 91-112.

[25] S. Romaguera and M. Schellekens, Partial metric monoids and semivaluation spaces, Topology Appl. 153 (2005), 948-962.

[26] M. Schellekens, The Smyth completion: a common foundation for denotational semantics and complexity analysis, in: Proc. MFPS 11, Electronic Notes in Theoretical Computer Science, vol. 1 (1995), URL: http://www.elsevier.nl/locate/entcs/volume1.html

[27] M. Schellekens, The correspondence between partial metrics and semivaluations, Theoret. Comput. Sci. 315 (2004), 135-149. 
[28] M. B. Sмyтh, Quasi-uniformities: Reconciling domains with metric spaces, in: Mathematical Foundations of Programming Language Semantics, 3rd Workshop, Tulane 1987, Lecture Notes Computer Science, vol. 298, M. Main et al (eds.), Springer, Berlin, 1988, 236-253.

[29] M. B. Sмyтh, Totally bounded spaces and compact ordered spaces as domains of computation, in: Topology and Category Theory in Computer Science, G.M. Reed, A.W. Roscoe and R.F. Wachter (eds.), Clarendon Press, Oxford 1991, 207-229. 